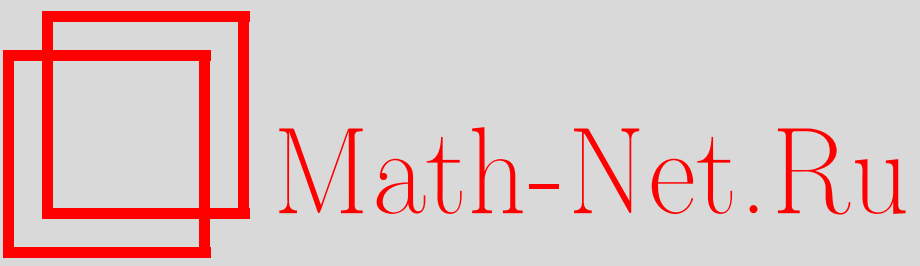

С. Х. Арансон, Е. В. Жужома, В. С. Медведев, И. А. Тельных, О двумерных базисных множествах грубых диффеоморфизмов трехмерных многообразий, УМН, 2000, том 55, выпуск 6, 123-124

DOI: https://doi.org/10.4213/rm336

Использование Общероссийского математического портала Math-Net.Ru подразумевает, что вы прочитали и согласны с пользовательским соглашением

http: //www.mathnet.ru/rus/agreement

Параметры загрузки:

IP : 54.209 .52 .79

26 апреля 2023 г., 12:44:23 


\title{
О ДВУМЕРНЫХ БАЗИСНЫХ МНОЖЕСТВАХ ГРУБЫХ ДИФФЕОМОРФИЗМОВ ТРЕХМЕРНЫХ МНОГООБРАЗИЙ
}

\author{
С. Х. АРАнСОН, Е.В. ЖУЖОмА, В. С. МЕДвЕДЕВ, И. А. ТЕЛЬнЫХ
}

Важным классом структурно устойчивых (грубых) диффеоморфизмов на замкнутых многообразиях $M^{n}$ являются диффеоморфизмы $А$ носова и структурно устойчивые $A$-диффеоморфизмы, введённые Смейлом. ${ }^{1}$

Пусть $\Omega \subset M^{n}$ - базисное множество коразмерности один (ориентируемое или неориентируемое). Для определенности, будем считать $\Omega$ аттрактором. Тогда достижимая извне граница множества $\Omega$ есть объединение конечного числа неустойчивых многообразий периодических точек аттрактора $\Omega$. Эта достижимая граница разбивается на попарно непересекающиеся связки [1], которые определяются следующим образом. Попарно различные неустойчивые многообразия $W^{u}\left(p_{i}\right)$ точек $p_{i} ;(i=1, \ldots, k)$ образуют $k$-связку, если существуют дуги $\left(x_{i}, y_{i}\right)^{s}$, принадлежашие одномерным устойчивым многообразиям, такие, что: 1$)\left(x_{i}, y_{i}\right)^{s} \cap \omega=\varnothing$; 2) $x_{i} \in W^{u}\left(p_{i}\right), y_{i} \in W^{u}\left(p_{i+1}\right)$ (здесь $\left.p_{k+1}=p_{1}\right)$; 3) число $k$-максимально возможное.

Известно [1], что на замкнутом или открытом $M^{n}(n \geqslant 3)$ базисные множества $\Omega$ коразмерности один могут содержать только 1-связки и 2-связки, при этом если в $\Omega$ есть хотя бы одна 1 -связка, то $\Omega$ неориентируемое, а если в $\Omega$ содержатся только 2-связки, то $\Omega$ ориентируемое.

Следующая теорема решает проблему о невозможности существования на замкнутых трехмерных многообразиях $M^{3}$ важных классов структурно устойчивых диффеоморфизмов.

Теорема. Пусть $f: M^{3} \rightarrow M^{3}$ - структурно устойчивый диффеоморфизм замкнутого трехмерного многообразия $M^{3}$. Тогда $f$ не мохет иметь двумерных базисных множеств с хотя бъ одной 1-связкой.

СХемА ДоКАЗАТЕЛЬСТвА. Предположим противное. Тогда из существования 1-связки вытекает, что $M^{3}$ содержит трехмерную область, гомеоморфную прямому произведению отрезка на лист Мёбиуса, и поэтому $M^{3}$ - неориентируемое многообразие. Рассмотрим замкнутое ориентируемое трехмерное многообразие $\widehat{M}^{3}$, являющееся для $M^{3}$ двулистным накрьвающим многообразием, и обозначим через $p$ проекцию $\widehat{M}^{3}$ на $M^{3}$. Тогда сушествует $A$-диффеоморфизм $\widehat{f}: \widehat{M}^{3} \rightarrow \widehat{M}^{3}$, накрьвающий диффеоморфизм $f: M^{3} \rightarrow M^{3}$ такой, что в спектральном разложении диффеоморфизма $\widehat{f}$ имеется ориентируемое двумерное базисное множество $\widehat{\Omega}(\Omega=p(\widehat{\Omega}))$, которое также можно считать аттрактором. Поскольку $f$ структурно устойчив и, следовательно, является $A$-диффееморфизмом и удовлетворяет сильному условию трансверсальности (R. Mane, C. Robinson), и так как $p: \widehat{M}^{3} \rightarrow M^{3}$ - локальный диффеоморфизм, то $\widehat{f}$ также структурно устойчив.

По условию, у структурно устойчивого диффеоморфизма $f$ существует открытая компонента $\sigma \subset M^{3} \downarrow \Omega$, в достижимую границу которой входит 1 -связка $W^{u}\left(m_{0}\right) \subset \Omega$, являющаяся неустойчивым двумерным многообразием периодической точки $m_{0} \in \Omega$. Не уменьшая общности, можно считать $m_{0}$ неподвижной точкой (она является граничной в смысле терминологии [1])

Возьмем произвольное поднятие $\widehat{m}_{1} \in \widehat{\Omega}$ точки $m_{0}$. Поскольку $\widehat{M}^{3}$ замкнутое и ориентируемое, то $\widehat{\Omega}$ имеет толко 2-связки. Поэтому существует еще ровно одна отличная от $\widehat{m}_{1}$ граничная периодическая точка $\widehat{m}_{2} \in \widehat{\Omega}$ такая, что $p\left(\widehat{m}_{1}\right)=p\left(\widehat{m}_{2}\right)=m_{0}$.

В силу [2], для структурно устойчивых диффеоморфизмов с базисными множествами коразмерности один, заданных на замкнутых $M^{n}(n \geqslant 3)$, и, в частности, для $\widehat{f}$ на $\widehat{M}^{3}$ выполняется,

Авторы благодарят РФФИ (грант № 99-01-00230) и INTAS (грант № 97-1843) за финансовую поддержку.

${ }^{1}$ Здесь и в дальнейшем, если не оговорено отдельно, за всеми определениями и понятиями, введенными различными авторами и используемыми в этой статье, отсылаем читателя к монографии [1]. 
что $W_{\phi}^{s}\left(\widehat{m}_{1}\right)$ и $W_{\phi}^{s}\left(\widehat{m}_{2}\right)$ принадлежат, соответственно, трехмерным неустойчивым многообразиям $W^{u}\left(\widehat{\alpha}_{1}\right), W^{u}\left(\widehat{\alpha}_{k+1}\right)(k$ - неотрицательное целое число, зависимое от $\widehat{f})$ отталкивающих периодических точек $\widehat{\alpha}_{1}, \widehat{\alpha}_{k+1}$, принадлежащих области $\widehat{\sigma} \subset \widehat{M}^{3} \downarrow \widehat{\Omega}^{2}$ Кроме того, в области $\widehat{\sigma}$ существуют еще отталкивающие периодические точки $\widehat{\alpha}_{i}(i=2, \ldots, k)$ с трехмерными неустойчивыми многообразиями и периодические точки $P_{i}(i=1, \ldots, k)$ с двумерными неустойчивыми многообразиями, которые удовлетворяют следуюшим условиям: 1) множество

$$
a_{12}=\left\{\widehat{m}_{1}\right\} \cup W_{\phi}^{s}\left(\widehat{m}_{1}\right) \cup \widehat{\alpha}_{1} \cup W^{s}\left(P_{1}\right) \cup \bigcup_{i=1}^{k}\left(P_{i} \cup \widehat{\alpha}_{i+1}\right) \cup W_{\phi}^{s}\left(\widehat{m}_{2}\right) \cup\left\{\widehat{m}_{2}\right\}
$$

есть простая замкнутая дуга с концевыми точками $\widehat{m}_{1}$ и $\left.\widehat{m}_{2} ; 2\right)$ на $a_{12}$ периодические точки $\widehat{\alpha}_{i}$; чередуются с периодическими точками $P_{i}$ (включая точки $\widehat{m}_{1}$ и $\widehat{m}_{2}$, размерность неустойчивых многообразий у которых равна двум), и, следовательно, число периодических точек на дуге $a_{12}$ нечетно.

Отсюда и из соотношений $p \widehat{f}=f p, p(\widehat{\Omega})=\Omega, p\left(\widehat{m}_{1}\right)=p\left(\widehat{m}_{2}\right)=m_{0}$ получаем следующие свойства: 1) $p\left(W_{\phi}^{s}\left(\widehat{m}_{1}\right)\right)=p\left(W_{\phi}^{s}\left(\widehat{m}_{2}\right)\right), p\left(\widehat{\alpha}_{i}\right)=p\left(\widehat{\alpha}_{k+2-i}\right), p\left(W^{s}\left(P_{j}\right)\right)=p\left(W^{s}\left(P_{k+1-j}\right)\right)$, $\left.p\left(P_{j}\right)=p\left(P_{k+1-j}\right)(i=1, \ldots, k+1, j=1, \ldots, k) ; 2\right)$ либо среди периодических точек $\widehat{\alpha}_{i}$ существует периодическая точка $\widehat{\alpha}_{\nu}$ с $p\left(W^{s}\left(P_{\nu-1}\right)\right)=p\left(W^{s}\left(P_{\nu}\right)\right)$ (в этом случае $k$ - четное, $\nu=\frac{1}{2}(k+2)$ и $\left.\left.p^{-1}\left(p\left(\widehat{\alpha}_{\nu}\right)\right)=\widehat{\alpha}_{\nu}\right) ; 3\right)$ либо среди периодических точек $P_{i}$ существует периодическая точка $P_{\nu}$ такая, что обе компоненты множества $W^{s}\left(P_{\nu}\right) \downarrow P_{\nu}$ отображается под действием $p$ в одну компоненту множества $W^{s}\left(p\left(P_{\nu}\right)\right) \downarrow p\left(P_{\nu}\right)$ (в этом случае $k$ - нечетное, $\nu=\frac{1}{2}(k+1)$ и $\left.p^{-1}\left(p\left(P_{\nu}\right)\right)=P_{\nu}\right)$.

В случае 2) в окрестности точки $\widehat{\alpha}_{\nu}$, а случае 3 ) в окрестности точки $P_{\nu}$ отображение $p: \widehat{M}^{3} \rightarrow$ $M^{3}$ не является локальным гомеоморфизмом, что невозможно.

Авторы благодарят Д. В. Аносова, В. З. Гринеса, М. И. Малкина за полезные замечания.

\section{СПИСОК ЛИТЕРАТУРЫ}

[1] Арансон С. Х., Аносов Д. В., Гринес В. З., Плыкин Р. В, Сатаев Е. А., Сафонов А. $\Phi$, Солодов В.В, Старков А.Н, Стёпин А. М, Шлячков С. В. Динамические системы с гиперболическим поведением // Итоги науки и техн. Совр. пробл. матем. Фундам. напр. Т. 66. М.: ВИНИТИ, 1991. С. 1-247. [2] Гринес В. З., Ж Кужома Е. В. Структурно устойчивые дифффеоморфизмы с ручными базисными множествами коразмерности один (в печати).

Нижегородский государственный технический университет; НИИ прикладной математики и кибернетики при Нижегородском

Принято редколлегией государственном университете им. Н.И. Лобачевского

03.10 .2000

\footnotetext{
${ }^{2}$ Здесь через $W_{\phi}^{s}\left(\widehat{m}_{i}\right)(i=1,2)$ обозначена та из компонент множества $W^{s}\left(\widehat{m}_{i}\right) \downarrow \widehat{m}_{i}$, которая не пересекается с $\widehat{\Omega}$.
} 